\title{
Morphodynamique dans la zone de swash sur la plage d'Egmond (Pays-Bas)
}

\author{
Christine DEGRYSE Doctorante (1), \\ Franck LEVOY Maître de conférences (1), \\ Gerben RUESSINK Docteur (2), \\ Claude LARSONNEUR Professeur (1)
}

(1) Université de CAEN - Unité «Morphodynamique Continentale et Côtière »UPRES-A CNRS 6143 - 24 rue des tilleuls - 14002 CAEN

(2) Université d'Utrecht - Institute for Marine and Atmospheric Research - Department of Physical Geography - P.O. Box 80115 3508 TC UTRECHT-Pays-Bas

\section{Résumé}

La morphodynamique dans la zone de swash est encore peu étudiée du fait des difficultés de mise en place d'appareils de mesure qualifiés. L'étude des barres de swash, leurs mécanismes de formation, de déplacement et de destruction est d'ailleurs peu décrit dans la littérature. Cette étude présente le comportement morphologique d'une barre de bas estran sur la plage d'Egmond aux Pays-Bas. Des données hydrodynamiques y sont associées. Une dynamique d'accrétion, avec la formation et la migration de la barre sableuse est d'abord identifiée. Celle-ci est liée à l'existence de vitesses orbitales élevées dans le domaine infragravitaire (fréquence inférieure à $0,04 \mathrm{~Hz}$ ). Une dynamique d'érosion est ensuite constatée avec un départ ou une redistributioñ du sédiment dans le profil correspondant à de faibles vitesses infragravitaires. Les données morphologiques acquises pendant le processus de swash confirme qu'il s'agit d'une barre strictement liée à cette dynamique se déplaçant préférentiellement en début de marée montante.

\begin{abstract}
The morphodynamic processes in the swash zone are still very poorly understood, mainly because of the difficulty to obtain field data. Mechanisms of formation, migration and erosion of swash bars need to be largely investigated. The purpose of this study is to observe the movement of a lower beach bar and the associated hydrodynamics in Egmond, Netherlands. The accretion and migration happen during high infragravitary velocity, while erosion of the bar takes place when low velocities of the infragravitary field are measured. Morphological data obtained during the swash processes reveal that this bar is a swash bar which moves mainly during the beginning of the flood period.
\end{abstract}




\section{Introduction}

La plupart des auteurs s'accordent sur l'importance de la zone de swash dans la morphodynamique des plages. Les processus hydrodynamiques spécifiques dans le swash conditionnent le sens des mouvements sédimentaires, orienté vers le trait de côte où il peut y avoir dépôt, ou vers la zone de surf interne, le sédiment étant potentiellement dispersé au large (Osborne et Rooker, 1997). Ainsi, les oscillations du swash (jet de rive - nappe de retrait) jouent un rôle significatif dans la genèse de la morphologie des plages. Les données de terrain concernant la zone de swash restent cependant peu nombreuses car difficiles à acquérir.

De multiples questions restent en suspens concernant l'évolution de la morphologie des plages sous l'effet du swash. Le sens et l'intensité des transports sédimentaires doivent être étudiés en fonction de la variabilité des conditions hydrodynamiques. Les paramètres qui influencent la morphodynamique dans la zone de swash sont donc à déterminer (Hughes et al., 1997 ; Baldock, 1997 ; Blewett et al., 1999). Des structures sédimentaires telles que les barres de swash sont sensées être engendrées par les processus propres à cette zone. Cependant, leur diversité, leur caractère parfois éphémère et la complexité de leur formation et de leur mobilité, méritent la réalisation d'investigations plus poussées sur le terrain.

\section{Caractéristiques du site étudié}

La plage d'Egmond (Pays-Bas) a été choisie comme site d'expérimentation dans le cadre du programme MAST III - COAST 3D pour réaliser une étude sur l'influence du swash sur les bas estrans microtidaux.

La côte hollandaise est caractérisée par un régime énergétique mixte, influencé à la fois par une dynamique de houle et de marée. Sur le site étudié, la houle dominante est de secteur SE à NW, avec une hauteur significative moyenne annuelle de $1,1 \mathrm{~m}$. La période moyennne est de cinq secondes. La marée est de type semi-diurne asymétrique, le flot a une durée de quatre heures et le jusant de huit heures. Le régime est microtidal, le marnage étant de $1,4 \mathrm{~m}$ en morte-eau, et de 2 $m$ environ en vive-eau. Lors de tempêtes, l'élévation du niveau marin à la côte, est parfois plus importante que le marnage.

La morphologie de l'avant-côte montre deux barres subtidales: une barre externe rectiligne et une barre interne prenant parfois une forme en croissant. La zone intertidale est relativement uniforme longitudinalement et s'étend transversalement sur environ une centaine de mètres, la pente de la plage variant de $0,5^{\circ}$ à $7,5^{\circ}$. La morphologie se caractérise par la présence fréquente de barres en parties basse et haute de la plage. Ces barres ont été décrites respectivement comme étant des barres de swash de marée basse et de marée haute (Kroon A., 1994). Elles sont parallèles à la côte, le plus souvent linéaires, mais présentent parfois des variations telles que des formes en croissant, ou d'autres liées à la présence de «rip channels ». La granulométrie des sables de plage varie entre 250 et $350 \mu \mathrm{m}$. 


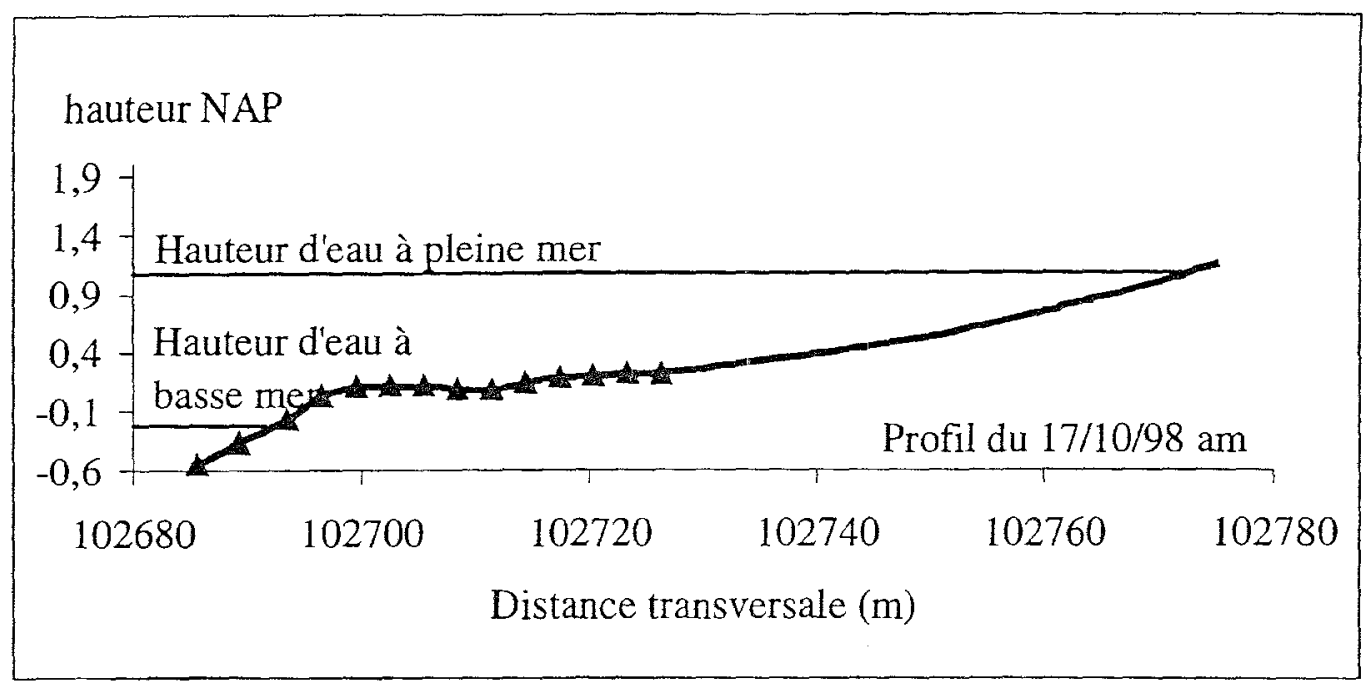

Figure 1 - Profil de la plage d'Egmond et localisation des repères topographiques Figure 1 - Egmond beach profile and localisation of the topography rods

\section{Méthodologie}

L'expérimentation in situ consiste à mesurer les changements altimétriques de l'estran au moyen de piquets disposés en ligne, en bas de plage, perpendiculairement à la côte (Fig. 1). Ces repères sont fixes et comportent des graduations. En plaçant une règle directement sur les différents piquets, et en s'assurant que sa base repose sur le sol, son sommet indique une hauteur, qui varie directement avec les fluctuations du fond sableux. Ainsi, il est possible de lire et d'enregistrer à un instant t la position du fond sédimentaire par rapport à celle mesurée à un temps $t_{0}$. La fréquence d'acquisition est de $5 \mathrm{mn}$ sur une période variant de $2 \mathrm{~h}$ à $8 \mathrm{~h}$, puis à chaque marée basse. La précision des mesures est de l'ordre de $0,5 \mathrm{~cm}$, voire $1 \mathrm{~cm}$ à proximité de la zone de surf. Les quatorze piquets, espacés de trois mètres, ont été positionnés en bas de plage, zone où le sable est en permanence saturé en eau.

Un modèle numérique de terrain a été levé de façon quotidienne ou biquotidienne au moyen d'un DGPS centimétrique. Cette mesure a permis de localiser les repères dans le système géodésique local (Fig.1).

Des mesures de courants et de houle ont été mesurées à environ $150 \mathrm{~m}$ au large du site d'expérimentation à proximité de la barre interne d'avant-côte. Des filtres passe-haut et passe-bas ont été utilisés pour séparer les vagues gravitaires et infragravitaires (respectivement de période inférieure ou supérieure à 25s)

Les données topographiques recueillies lors de huit marées basses consécutives du 15 octobre au 19 octobre 1998, sont présentées sur la figure 2 . La partie basse de l'estran présente une barre sableuse, objet de la présente étude. 


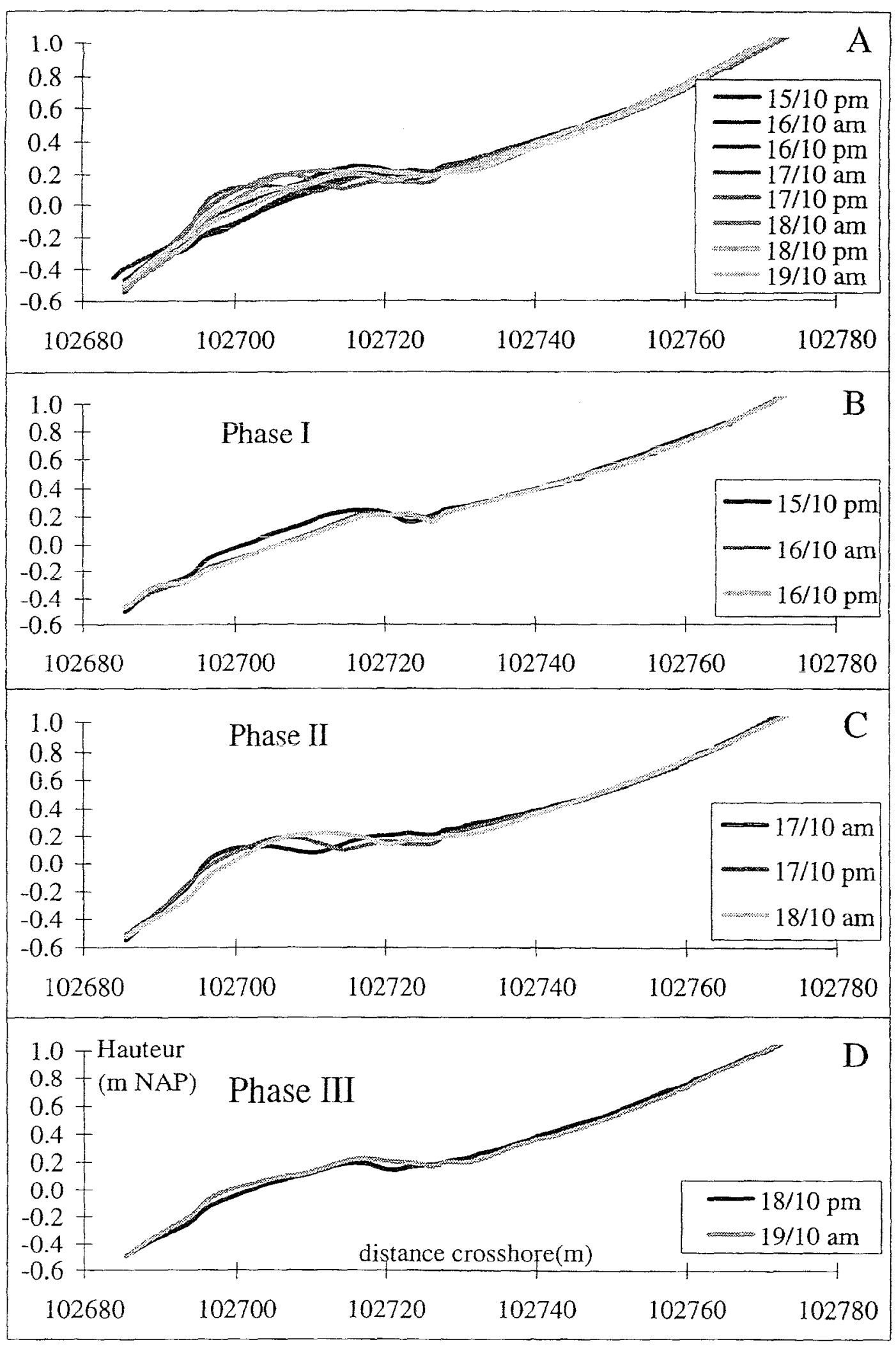

Figure 2: Variations topographiques observées à marée basse

Figure 2 : Topographic variations observed at low tide 


\section{Résultats}

\subsection{Observations des données morphologiques}

La totalité des profils montre une grande mobilité de la barre dont l'amplitude varie de quinze à quarante-cinq centimètres et la largeur de vingt-cinq à quarante mètres. La séquence de profils peut être divisée en trois phases successives se caractérisant par une évolution singulière (Fig. 2).

La première phase concerne les marées basses du 15 octobre dans l'aprèsmidi, et du 16 octobre le matin et l'après-midi (Fig. 2B). Le profil initial permet d'observer une barre de basse plage très dissymétrique d'une amplitude de trente centimètres. Le profil topographique levé lors de la marée basse suivante, permet d'observer un aplatissement de la barre avec un adoucissement de la pente du talus et une érosion significative du revers. La barre montre une légère migration vers le haut de plage, d'environ trois mètres. Cette morphologie n'évolue pas au cours de la marée suivante. Le calcul des cubatures déterminé sur le domaine de la basse plage confirme son érosion (Fig. 3).

En début de seconde phase, une barre d'amplitude égale à une trentaine de centimètres apparaît (Fig. 2C). Le volume sédimentaire de la basse plage a augmenté de façon relativement importante. La barre est très faiblement dissymétrique. Lors des deux marées consécutives le 17 et 18 Novembre, elle progresse de cinq à six mètres vers le haut de plage et devient fortement dissymétrique, avec un talus dirigé vers la côte. Le volume sédimentaire du bas estran est conservé pendant les trois cycles tidaux.

Enfin, la troisième phase montre une forte diminution de l'amplitude de la barre par rapport à celle mesurée dans la seconde phase (Fig.2D). Cette érosion est confirmée par l'évolution du volume sédimentaire de la basse plage (Fig. 3). Cette dernière phase est marquée par une très faible progression du talus vers la côte accompagnée d'une faible augmentation de l'amplitude de la barre.

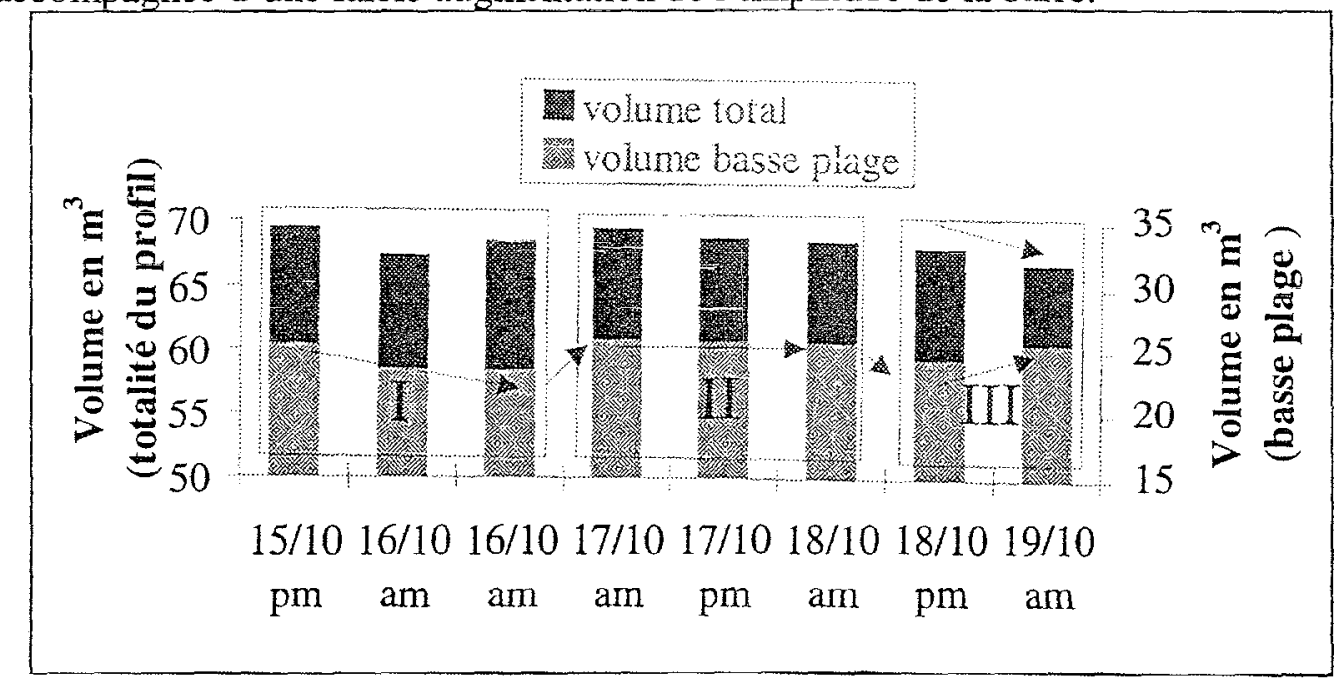

Figure 3: Variations de volume de la basse plage et de la totalité du profil, par mètre linéaire

Figure 3: Variations of the beach volume and of the lower beach volume. 
Pour résumer, les trois phases montrent deux dynamiques différentes. Lors de la première et de la troisième période, la tendance est à l'érosion, avec aplanissement de la structure préexistante et départ de matériel. La seconde phase montre une dynamique de construction et de déplacement de barre. Le sédiment est apporté et conservé sur le bas de plage.

\subsection{Observation des données hydrodynamiques}

Kroon (1994) attribue les changements morphologiques des barres de swash qu'il a étudiées à l'amplitude des vagues incidentes et à la hauteur d'eau maximale atteinte à marée haute. Dans le cas présent, l'amplitude de la houle est en phase avec les variations de hauteur du plan d'eau statique. Cependant, ni la hauteur du plan d'eau statique à marée haute, ni les hauteurs des vagues incidentes et leurs vitesses orbitales associées ne montrent de variations qui puissent être corrélables avec les trois phases morphodynamiques observées.

De nombreux auteurs soulignent l'importance dans la zone de swash des vagues de période supérieure à $25 \mathrm{~s}$, dites vagues infragravitaires. Leurs vitesses et leurs amplitudes y sont en effet maximales (Beach et Steinberg 1991, Osborn et Rooker, 1999). Sur la figure 4 sont représentées les variations de hauteurs du plan d'eau associées aux vitesses des infragravitaires. Trois dynamiques semblent s'identifier : Une première période de vitesses qui décroissent de 25 à $8 \mathrm{~cm} / \mathrm{s}$. Une rapide augmentation est ensuite constatée entre le 16 et le 17 Octobre les vitesses restent alors assez élevées, variant entre 17 et $30 \mathrm{~cm} / \mathrm{s}$. Entre le 18 et le 19 Octobre, de faibles vitesses, atteignant moins de $10 \mathrm{~cm} / \mathrm{s}$, sont constatées.

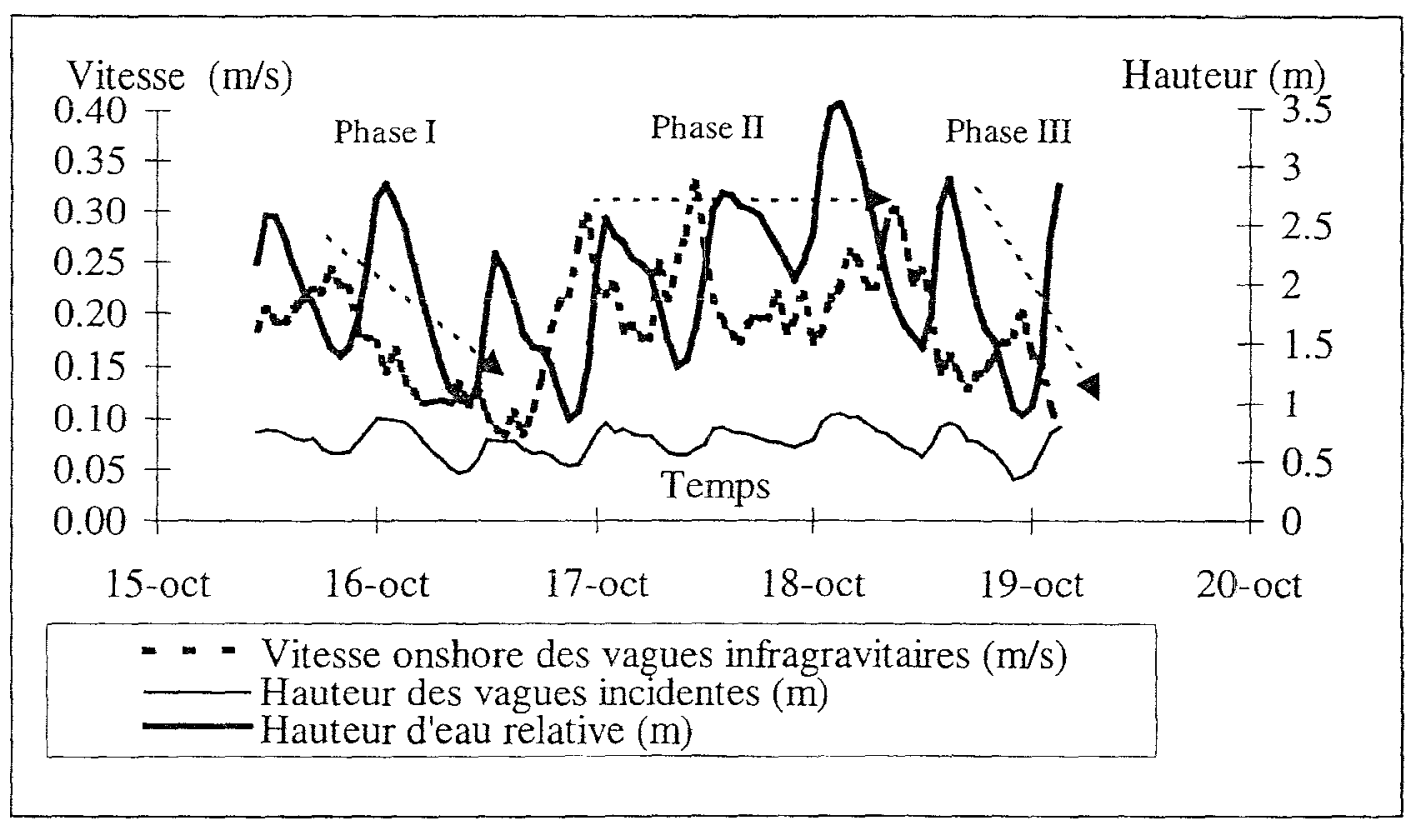

Figure 4 : Données de houle et variations du plan d'eau statique Figure 4 : Waves caracteristics and water level 
Ces trois phases correspondent exactement à celles identifiées lors des observations morphologiques. La construction de la barre se produit quand les vagues infragravitaires génèrent des vitesses orbitales élevées. Le déplacement de la barre décrit sur la figure $2 \mathrm{C}$ est probablement imputable à l'augmentation de la hauteur du plan d'eau statique au cours de marées successives (Fig. 4) tel que l'a décrit Kroon (1994). Cependant, il apparaît clairement que cette migration n'est possible qu'en présence d'une énergie infragravitaire élevée. Avec des vitesses moindres, la morphologie de la barre n'est pas conservée. Il y a aplanissement général du profil de plage, soit par départ, soit par redistribution des sédiments sur le profil. Contrairement aux vagues incidentes, les vagues infragravitaires ne sont pas en phase avec les courbes de marée (Fig. 4). Les pics de vitesse s'observent en début de marée montante. Les variations morphologiques les plus importantes de la barre se produisent probablement lors du passage du swash sur le bas estran.

\subsection{Observations des variations morphologiques lors de la marée montante}

L'acquisition de profils topographiques à une cadence de cinq minutes permet d'observer le déplacement de la barre de bas de plage sous l'influence stricte du swash (Fig. 5). La migration vers la côte de cette barre se fait par l'érosion progressive de son revers et accrétion du talus. Le sédiment semble être déplacé du flanc le moins pentu de la barre pour être déposé sur son flanc raide. Kroon (1994) décrit qualitativement le processus de migration de ce type de barre et conclut que le développement de la barre s'observe pendant les périodes de flot. Ceci est en accord avec les observations faites le 17 Octobre 1998 (Fig. 5). Il a été impossible de suivre les variations topographiques à marée haute et marée descendante. Cependant, le profil levé à marée basse, en pointillé sur la figure, ne montre qu'une faible accrétion du revers de la barre. Cet engraissement est probablement induit par un apport de sédiments lors des processus de run-up de marée descendante (Kroon 1994). La majorité du déplacement s'est donc effectivement produite en début de marée montante, quand l'énergie des vagues infragravitaire est maximale.

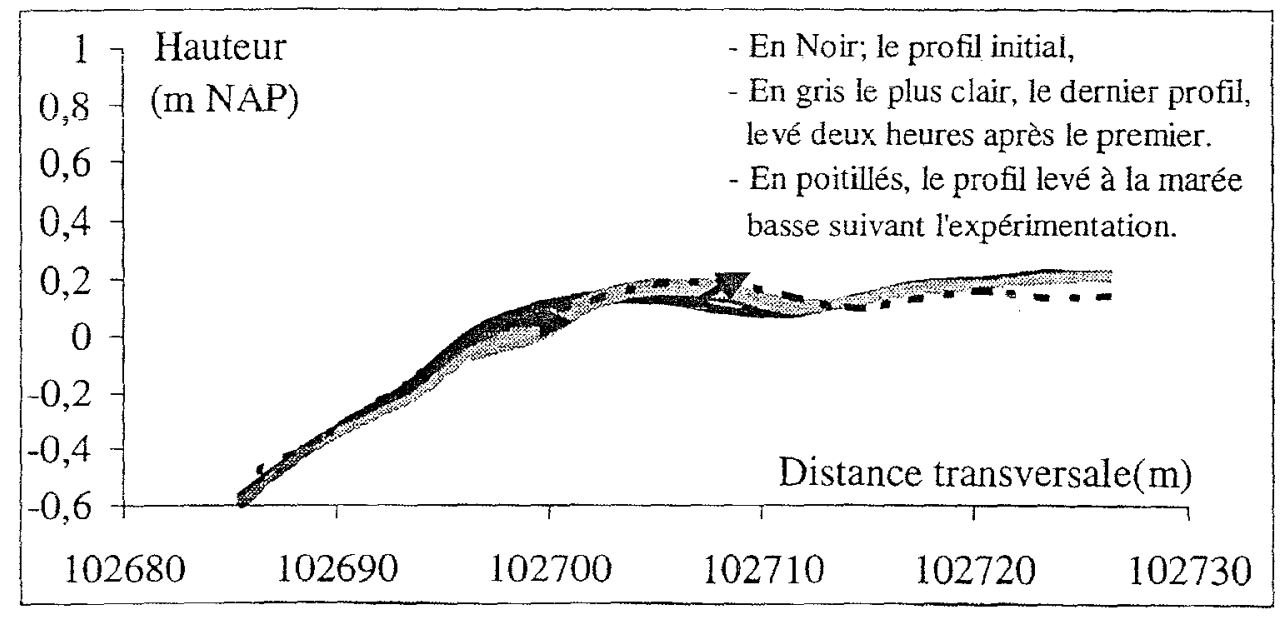

Figure 5: Succession des profils topographiques levés au cours du swash Figure 5: Topographic profiles succession obtained during swash processes 


\section{Conclusion}

Au regard des huit cycles de marée étudiées, la barre sableuse observée en partie basse de la plage d'Egmond semble répondre rapidement aux changements hydrodynamiques et notamment aux variations de vitesses orbitales induites par des vagues infragravitaires (de fréquence inférieure à $0,04 \mathrm{~Hz}$ ). Avec des vitesses élevées, la formation d'une barre est observable en partie basse de la plage. Cette barre est alors conservée, puis peut migrer vers la côte en période de revif à la faveur d'un cycle morte-eau / vive-eau. La majorité des changements morphologiques se fait en début de marée montante, alors que les vitesses infragravitaires sont maximales et lorsque le processus de swash agit sur la basse plage. En phase de décroissance des vitesses infragravitaires, il y a une érosion de la crête de la barre de swash et un aplanissement général du profil, soit par départ de matériel ou/et par redistribution du sédiment au sein du profil.

\section{Bibliographie}

Baldock T.E., Holmes P., et Horn D.P., 1997. Low frequency swash motion induced by wave grouping. Coastal Engineering, 32. 197-222.

Beach R.A. et Sternberg R.W., 1991. Infragravity driven suspended sediment transport in the swash, inner and outer surf zone. Coastal sediments '91, New York: American Society of civil Enginners, 114-128.

Blewett J.C., Holmes P., et Horn D.P., 1999. Measurement and modelling of swash hydrodynamics. Coastal sediments '99. New York: American Society of civil Engineers, 377-392.

Hughes M.G., Masselink G., et Brander R.W., 1997. Flow velocity and sédiment transport in the swash zone of a steep beach. Marine Geology, 138, 91-103.

Kroon, A., 1994. Sediment-transport and Morphodynamics of the Beach and Nearshore Zone near Egmond, The Netherlands, PhD-Thesis, Utrecht University, 275pp.

Osborn P.D., et Rooker G.A., 1999. Sand Re-Suspension Events in a High Energy Infragravity Swash Zone. Journal of Coastal Research, 15/1, 74-86.

\section{Remerciements:}

Le projet MAST III COAST3D est financé par l'Union Européenne, le « Rijkswaterstaat», le «UK Ministry of Agriculture Fisheries and Food » et le «UK Natural Environment Research Council ». Les données de terrain n'auraient pu être obtenues sans la collaboration efficace de nos collaborateurs, et notamment les membres de l'équipe du GRESARC, qu'ils en soient chaleureusement remerciés. 